\title{
ADSORPTION EFFECTS IN LIGHT-INDUCED DRIFT
}

\author{
G. NIENHUIS \\ Huygens Laboratory, University of Leiden, P.O. Box 9504, 2300 RA Leiden, The Netherlands \\ and Physics Laboratory, University of Utrecht, P.O. Box 80 000, 3508 TA Utrecht, The Netherlands
}

Received 8 December 1986

\begin{abstract}
We describe the effect of light-induced drift, while allowing for adsorption of the active atoms on the wall of the cell. Assuming a rapid rate of adsorption and desorption, we express this effect by a modification of the diffusion equation for the volume density in the boundary layer near the wall. Explicit solutions are discussed in opposite special cases, corresponding to a large or a small mean free path of the active atoms.
\end{abstract}

\section{Introduction}

Light-induced drift (LID) can occur when optically active atoms immersed in a buffer gas are excited in a velocity-selective fashion, so that the excited atoms have a nonzero preferential velocity $[1,2]$. The effect arises from a difference in thermalization rate of the velocity distribution for both states of the atoms, due to different cross sections for elastic collisions with buffer-gas particles. In the steady state, this leads to a flow of excited atoms that is not exactly counterbalanced by an opposite flow of ground-state atoms, and a net flow of the atoms remains. This net flow of atoms can set up a density gradient in a closed cell [3]. For an optically thick system, where the intensity of the light passing through the medium can display a strong variation in the propation direction, this density gradient can become dramatic. The light can effectively sweep away the atoms towards the dark end of the cell, while leaving the buffer-gas particles untouched. This piston action of light, which was predicted in ref. [2], has recently been demonstrated for $\mathrm{Na}$ atoms in argon $[4,5]$. However, the obscrved drift velocity in these experiments was appreciably lower than predicted by simple gas-kinetic considerations. This difference was attributed to the effect of adsorption of the sodium atoms on the wall of the capillary vapor cell.

In the present paper, we study the effect of adsorption and desorption in more detail. The effect is expressed by a modification of the diffusion equation for the atomic density in the boundary layer near the wall. We specify solutions of this modified diffusion equation in some cases of practical interest.

\section{Diffusion equation and absorption equation}

First we recall some results from a theoretical description of LTD. The flow of active atoms $j(r, t)$, irrespective of their state, can be put in the form

$j(r, t)=-D \operatorname{grad} n(r, t)+u(r, t) n(r, t)$,

where $n$ is the volume density of active atoms. The first term on the righthand side of (1) is the ordinary diffusive flow, with $D$ the diffusion constant for ground-state atoms in the buffer gas. The second term is the light-induced flow with $\boldsymbol{u}$ the drift velocity, defined as the average velocity of the active atoms at position $r$ and time $t$. A treatment based upon the separation of time scales for the radiative transitions and for the macroscopic drift effect leads to an expression for the drift velocity $u$ as a function of the light intensity $I(r, t)$ at the same position and time, for a given light frequency. The drift velocity is directed along the propagation direction of the light, which we take as the $z$-direction. When the saturated homogeneous linewidth is small compared with the Doppler width, the drift velocity obeys the equality $[6,7]$ 
$u n=-D q \partial I / \partial z$,

when the parameter $q$ is defined by the equality

$D q=-\left(\kappa^{\prime}-\kappa\right) v_{0} /[\kappa \hbar \omega(A+\kappa)]$

in terms of the thermalization rates $\kappa^{\prime}$ and $\kappa$ for excited atoms and ground-state atoms, and the spontaneous decay rate $A$. The Doppler selected velocity $v_{0}=\left(\omega-\omega_{0}\right) / k$ is nonzero only when the light frequency $\omega$ is off-resonance from the transition frequency $\omega_{0}$. The intensity variation follows from the absorption equation [7]

$\partial I / \partial z=-n f(I)$,

where $f$ is a function of the intensity $I$. Without saturation, we have $f(I)=Q I$, with $Q$ the absorption cross section, that is proportional to the Maxwell distribution at the selected velocity $v_{0}$. Explicit expressions for the saturation behavior of $f(I)$ at higher values of $I$ are discussed in ref. [7].

The diffusion equation for the atomic density $n$ follows if we substitute (1) into the continuity equation

$\partial n(\boldsymbol{r}, t) / \partial t=-\operatorname{div} \boldsymbol{j}(\boldsymbol{r}, t)$.

The diffusion equation for the density, together with the absorption equation (4) constitutes a pair of coupled differential equations. Explicit solutions have been obtained in special cases. One such case arises in the stationary case, where both $I$ and $n$ are independent of time. Another solution of practical interest is the solitary density packet, with a fixed shape, and moving with a uniform velocity $w$. In this case, both $n$ and $I$ are a function of $z-w t$ alone [6, $7]$. Both these special solutions are one-dimensional in the sense that $n$ and $I$ do not vary with $x$ and $y$, but solely with the $z$ coordinate indicating the position along the propagation direction of the light.

\section{Surface density and volume density}

A position $r$ within the volume of the vapor cell can be specified by the distance $\rho$ to the nearest point on the wall, and the two-dimensional coordinate $\sigma$ indicating that point on the wall. We model the process of adsorption and desorption by adopting two closely related assumptions. First we consider an atom adsorbed to the wall at the position denoted by the wall coordinate $\sigma$. We assume that at desorption, the atom is deposited in the volume at the position $\boldsymbol{r}=(\rho, \sigma)$ with a probability distribution $b(\rho)$ over the distance to the wall. By definition, this distribution is normalized, and we write

$\int_{0}^{\infty} \mathrm{d} \rho b(\rho)=1$,

provided that the range of $b$ is small compared with the smallest dimension of the cell. The second assumption is that the adsorption rate of an atom in the volume at a distance $\rho$ from the wall is proportional to this same function $b(\rho)$. As one easily checks, this second assumption follows from the first one if we require that in adsorption-desorption equilibrium the volume density $n$ is constant as a function of $\rho$ in the boundary layer near the wall. A natural assumption for the distribution $b(\rho)$ would be the exponential function

$b(\rho)=\lambda^{-1} \exp (-\rho / \lambda)$,

with $\lambda$ the free path of an atom in the buffer gas.

With these two assumptions, we can write down the coupled rate equations for the volume density $n(\boldsymbol{r})$ and the surface density $\nu(\sigma)$. For simplicity we suppress the surface coordinate $\sigma$, and we indicate explicitly only the distance $\rho$ from the surface. When the desorption rate of an atom adsorbed at the wall is denoted as $\lambda$, the rate equations take the form

$(\partial / \partial t) \nu=-\gamma \nu+\alpha \gamma \bar{n}$

$(\partial / \partial t) n(\rho)=\gamma b(\rho) \nu$

$$
-\alpha \gamma b(\rho) n(\rho)-\operatorname{div} \boldsymbol{j}(\rho),
$$

with

$\bar{n}=\int \mathrm{d} \rho b(\rho) n(\rho)$,

a weighted average of the volume density in the boundary layer near the surface point $\sigma$. The particle flow $j$ in the last term in (9) is defined in eq. (1). This last term is the rate of change of the density $n$ due to diffusion and light-induced drift. The other terms in (8) and (9) represent adsorption and desorption. The parameter $\alpha$ is a proportionality 
constant which has the physical significance of the equilibrium ratio between the surface density $\nu$ and the volume density $n$. This also means that

$\alpha \gamma=p v_{0} / 4$,

with $v_{0}$ the average thermal velocity of an atom, and $p$ the sticking probability for an atom that hits the wall.

The effect of adsorption on LTD must be distinguished from the wall effects discussed in refs. [8] and [9]. In that work the wall effectively contributes to the LID effect, by a difference in the velocity thermalization for excited atoms and ground-state atoms at a wall collision. In the present paper, the adsorption to the wall temporarily removes atoms from the interaction with the light field, thereby affecting the LID effect produced by the buffer gas.

\section{Separation of time scales}

We now adopt the simplifying assumption that the time scale $\gamma^{-1}$, characteristic for the approach to adsorption-desorption equilibrium is rapid, so that during time lapses of this order the macroscopic flow due to diffusion and light-induced drift has had no time to cause any appreciable change in the density. Then we may assume that the rapid change described by the terms proportional to $\gamma$ in (8) and (9) drive $n$ and $\nu$ to their steady-state ratio, determined by these terms. This means that after a rapid transient of the order of $\gamma^{-1}, \nu$ and $n$ have the ratio

$\alpha \bar{n}=\nu=\alpha n(\rho)$,

at least for values of $\rho$ within the boundary region where $b(\rho)$ is not negligible. This result may be substituted into any equation that contains exclusively the slow evolution. Such an equation may be constructed by taking the linear combination of (8) and (9) in the form

$$
\begin{aligned}
& (\partial / \partial t)[n(\rho)+b(\rho) \nu] \\
& \quad=-\alpha \gamma b(\rho)[n(\rho)-\bar{n}]-\operatorname{div} j(\rho) .
\end{aligned}
$$

Note that the first term on the right-band side of (13) still contains the rapid rate $\gamma$, but it is multiplied by the deviation $n-\bar{n}$ of the density from its average in the boundary layer, which is a small quantity. If we now substitute eq. (12) into (13), we arrive at the approximate evolution equation

$$
(\partial / \partial t)[1+\alpha b(\rho)] n(\rho)=-\operatorname{div} \boldsymbol{j}(\rho)
$$

for the volume density alone. The surface density $\nu$ is effectively eliminated, since it is assumed to follow the variations of the volume density $n$ in the boundary layer in an adiabatic way.

Eq. (14) is the basic result of this paper. It demonstrates that the adsorption to the wall affects the equation of motion for the volume density in the boundary layer. Outside this layer, $b(\rho)$ is zero, and (14) reduces to (5). In order to illustrate the significance of (14), we consider two opposite special cases.

\section{Large free path}

When the free path $\lambda$ of the atoms is large compared with the diameter of the cell, then each desorbed atom is deposited homogeneously distributed over the cross section of the cell, and $b$ may be considered as a constant. This means that each position in the cell is within the boundary layer. Since according to eq. (9) the parameter $\alpha \gamma b$ is the rate of adsorption of an atom in the volume, while $\gamma$ is the desorption rate, we may conclude that $\alpha b$ is the ratio of the number of atoms adsorbed to the wall and the number of atoms in the volume of the cell. Then eq. (14) may be written as

$$
(\partial / \partial t) n=-s \operatorname{div} \boldsymbol{j}
$$

with $s=(1+\alpha b)^{-1}$ the fraction of atoms within the volume of the cell. This illustrates that in this case of a large free path, the effect of adsorption is simply to slow down the evolution of the density by a factor $s$. This is quite understandable, since at any instant only a fraction $s$ of the atoms participate actively in the process of diffusion and drift. A similar result has already been discussed in ref. [5].

\section{Small free path}

Now we assume that the free path $\lambda$ is small compared with the diameter of the cell. Then we may separate the volume in a small boundary layer of thickness a few times $\lambda$, and the remaining volume of 
the cell. In the boundary layer, adsorption-desorption processes dominate, and drive the density $n(\rho)$ to a constant value, while fixing the ratio $\nu / n$ to the value $\alpha$. As viewed from the main volume of the cell, the normalized distribution $b(\rho)$ is very narrow, and may be considered as a delta function. Outside this narrow boundary layer, eq. (5) determines the evolution of the density, with the atomic flow given in (1). A change in $n$ at the boundary is accompanied by a corresponding change in the surface density $\nu=\alpha n$. This change in the surface density must be reflected in a nonzero value of the normal component of the atom flow $j$ orthogonal to the wall surface. We find

$j_{t}=-\alpha \partial n / \partial t$

where $j_{t}$ is the component of the flow (1) at the wall, in the direction perpendicular to the wall, and pointing inwards. This equation may be viewed as a singular part of the continuity equation, with $\nu=\alpha n$ a density with a delta-function behavior in one direction. Obviously, eq. (16) imposes a boundary condition on the evolution equation for the volume density. When $\alpha=0$, no surface density arises, and we recover the usual boundary condition that orthogonal flow components must vanish. In conclusion, we notice that the effect of adsorption and desorption to the wall can be accounted for simply by imposing the boundary condition (16) to the solution of eqs. (5) and (1).

An explicit solution for a solitary density packet can be obtained, for the model case of a large flat boundary surface parallel to the propagation direction of the light. We take the propagation direction as the $z$-direction, and the wall as the $y z$-plane, so that the boundary condition (16) refers to the $x$-component of the flow. Generalizing the solitary-packet solution in one dimension [6,7], we look for a solution in which the density packet moves in the $z$ direction with a constant shape, and a uniform velocity $w$. In order to take care of the boundary condition (16), we allow for a linear displacement of the density packet with the $x$-value, as sketched in fig. 1 . Hence we look for a solution in the form

$n(x, z, t)=m(z-\eta x-w t)$

$I(x, z, t)=J(z-\eta x-w t)$ with $m$ and $J$ one-parameter functions. The diffusion equation (5) takes the form

$$
\begin{aligned}
- & w \mathrm{~d} m(z) / \mathrm{d} z=D\left(1+\eta^{2}\right) \mathrm{d}^{2} m(z) / \mathrm{d} z^{2} \\
& +D q \mathrm{~d}^{2} J(z) / \mathrm{d} z^{2}
\end{aligned}
$$

and the absorption equation (4) now reads

$\mathrm{d} J(z) / \mathrm{d} z=-m(z) f(J(z))$.

The boundary condition (16) gives the relation

$\eta=\alpha w / D$,

after using eq. (1), while noting that $\boldsymbol{u}$ is parallel to the $z$-direction, and therefore gives no contribution to $j_{t}$.

A solitary-packet solution is only possible for a finite optical thickness, and the function $m(z)$ must vanish in the limits $z \rightarrow \pm \infty$. The incident intensity $I_{0}$ must be equal to $J(z)$ for $z \rightarrow-\infty$. If we integrate eq. (19) twice, while introducing the integrated density

$M(z)=\int_{-\infty}^{z} \mathrm{~d} z^{\prime} m\left(z^{\prime}\right)$,

we obtain the equation

$-w M=D\left(1+\eta^{2}\right) \mathrm{d} M / \mathrm{d} z-D q\left[I_{0}-J(M)\right]$,

with

$\mathrm{d} J / \mathrm{d} M=-f(J)$.

For $z \rightarrow \infty$, the density $\mathrm{d} M / \mathrm{d} z$ must vanish, and we find the relation

$w=D q\left(I_{0}-I_{\infty}\right) / M_{\infty}$

between the packet velocity $w$, the incident intensity $I_{0}$, and the integrated density $M_{\infty}$, which is a measure for the optical thickness. The intensity $I_{\infty}=J(\infty)$ after passage through the packet is negligible for an optically thick system.

Eq. (25) is exactly the same as in the one-dimensional solution without wall effects $[6,7]$, so that the relation between packet velocity $w$ and optical thickness is unaffected by adsorption in this model case. On the other hand, the adsorption effect in eq. (23), as expressed by the presence of the factor $\eta^{2}$, indicates that the density distribution of the atoms is stretched in the $z$-direction by the factor $1+\eta^{2}$. As 


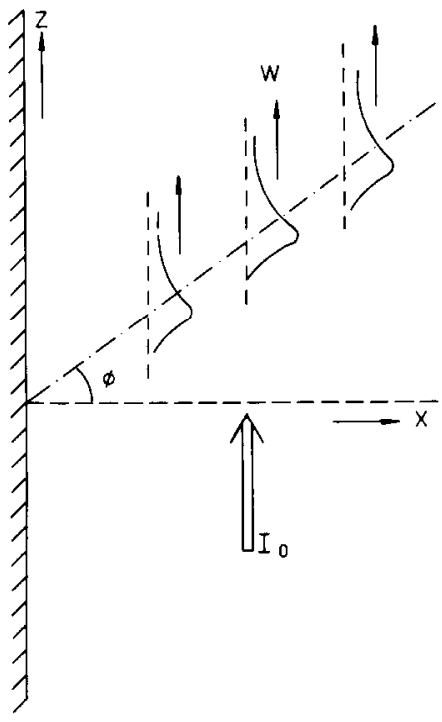

Fig. 1. Sketch of the density distribution $n$ as a function of $x$ and $z$ at a certain instant of time $t$. The angle $\phi$ denotes the angle of the line indicating the density maximum with the plane of the wall, and it is related to the parameter $\eta$ by eq. (26).

sketched in fig. 1 , the parameter $\eta$ obeys the relation $\eta=\tan \phi$,

with $\phi$ the angle between the line connecting the maxima of the density with the $x$-axis, normal to the boundary plane. The stretched factor $1+\eta^{2}$ is therefore equal to $\cos ^{-2} \phi$. The particle flow $j$ has a $z$ component

$$
\begin{aligned}
& j_{z}(x, z, t)=-D\left[m^{\prime}(z-\eta x-w t)\right. \\
& \left.\quad+q J^{\prime}(z-\eta x-w t)\right],
\end{aligned}
$$

with $m^{\prime}$ and $J^{\prime}$ the derivatives of $m$ and $J$. Moreover, the flow has an $x$-component given by

$j_{x}(x, z, t)=D \eta m^{\prime}(z-\eta x-w t)$,

which is needed to respect the continuity equation (5) in the present form (19).

\section{Conclusions}

We have demonstrated that the effect of adsorption to the wall on light-induced drift can be modelled in the form of eqs. (8) and (9) for the volume density $n$ and the surface density $\nu$. Assuming that the rate of adsorption and desorption is rapid compared with the rate of macroscopic flow, we obtain eq. (14) for the volume density $n$ alone. In the case of a long cell with a diameter that is small compared with the mean free path of the active atoms, the net result of adsorption is simply a slowing down of the time evolution by a factor $s$, that equals the fraction of atoms in the volume of the cell. When the mean free path is small compared with all dimensions of the cell, the adsorption effect gives rise to an effective boundary condition (16) for the component of the atom flow normal to the wall. For a solitary packet moving with uniform velocity $w$ this boundary condition gives rise to a skewness of the crest of the packet, expressed by the angle $\phi$ with respect to the normal direction to the wall. The angle $\phi$ is related by (26) and (21) to the packet velocity $w$, the diffusion constant $D$ and the ratio $\alpha$ of the surface density to the volume density.

\section{Acknowledgements}

The author gratefully acknowledges several illuminating discussions with F.Kh. Gel' mukhanov and with J.P. Woerdman.

\section{References}

[1] F.Kh. Gel'mukhanov and A.M. Shalagin, Pis'ma Zh.Eksp. Teor. Fiz. 29 (1979) 773 [JETP Letters 29 (1979) 711].

[2] F.Kh. Gel' mukhanov and A.M. Shalagin, Zh. Eksp. Teor. Fiz. 78 (1980) 1674 [Sov. Phys. JETP 51 (1980) 839].

[3] S.N. Atutov, P.L. Chapovsky and A.M. Shalagin, Optics Comm. 43 (1982) 265.

[4] H.G.C. Werij, J.P. Woerdman, J.J.M. Beenakker and I. Kuščer, Phys. Rev. Lett. 52 (1984) 2237.

[5] H.G.C. Werij, J.E.M. Haverkort and J.P. Woerdman, Phys. Rev. A 33 (1986) 3270.

[6] G. Nienhuis, Phys. Rev. A 31 (1985) 1636.

[7] G. Nienhuis, Phys. Rep. 138 (1986) 151.

[8] A.V. Ghiner, M.I. Stockmann and M.A. Vaksman, Phys. Lett. 96A (1983) 79.

[9] M.A. Vaksman and A.V. Gainer, Zh. Eksp. Teor. Fiz. 89 (1985) 41 [Sov. Phys. JETP 62 (1985) 23]. 